\title{
Selective removal of the (2-naphthyl)methyl protecting group in the presence of $p$-methoxybenzyl group by catalytic hydrogenation
}

\author{
László Lázár, ${ }^{a}$ Lóránt Jánossy, ${ }^{\text {a } M a g d o l n a ~ C s a ́ v a ́ s, ~}{ }^{a}$ Mihály Herczeg, ${ }^{a}$ Anikó Borbás ${ }^{\text {a,* }}$ and \\ Sándor Antus ${ }^{\mathrm{a}, \mathrm{c}}$ \\ ${ }^{a}$ Research Group for Carbohydrates of the Hungarian Academy of Sciences, University of \\ Debrecen, H-4010, Debrecen, PO Box 94, Hungary \\ ${ }^{c}$ Department of Organic Chemistry, University of Debrecen, H-4010, Debrecen, PO Box 20, \\ Hungary \\ E-mail: borbas.aniko@science.unideb.hu
}

\section{Dedicated to Prof. Ferenc Fülöp on the occasion of his 60th birthday}

\begin{abstract}
Selective cleavage of the (2-naphthyl)methyl (NAP) group in the presence of $p$-methoxybenzyl (PMB), benzyl and benzylidene groups was achieved by catalytic hydrogenation with a series of monosaccharides. At a disaccharide level, the PMB group was stable upon hydrogenolysis of the NAP, however, partial cleavage of benzyl ether functions was observed.
\end{abstract}

Keywords: Carbohydrates, orthogonal protecting groups, benzyl type protecting groups, selective removal, catalytic hydrogenation

\section{Introduction}

In carbohydrate chemistry, the benzyl-type ethers are the most frequently used protecting groups, since they are removable upon mild catalytic hydrogenation, and, due to their different reactivities, adoptable in orthogonal protecting strategies. ${ }^{1}$ The $p$-methoxybenzyl (PMB) ether can be cleaved selectively by DDQ (2,3-dichloro-5,6-dicyano-1,4-benzoquinone) ${ }^{2}$ or CAN (cerium(IV) ammonium nitrate $)^{3,4}$ leaving most of the protecting groups intact, therefore, it became one of the most popular tools for temporary hydroxyl protection. However, the PMB group has the disadvantage of extreme acid-sensitivity. The 2-(naphthyl)methyl (NAP) group, ${ }^{5,6}$ being removable by either catalytic hydrogenation ${ }^{6-8}$ or $\mathrm{DDQ}^{9}$ in the presence of benzyl ether and, unlike its $p$-methoxybenzyl counterpart, being stable under acidic conditions, seems to be a remarkably useful protecting group for polyhydroxy systems. 
Recently, Spencer et al. have demonstrated a sequential deprotection strategy for sugars based upon the PMB, NAP and benzyl groups, applying CAN as a selective reagent to cleave PMB in the presence of the NAP-ether. ${ }^{4}$ This set of benzyl-type protecting groups would be more useful, if orthogonal deprotection of NAP would be carried out in the presence of PMB.

Investigating the relative stability of benzyl, p-methoxybenzyl, 2-(naphthyl)methyl, 1(naphthyl)methyl, ${ }^{10,11}$ diphenylmethyl, 9-fluorenyl and $p$-chlorobenzyl ethers to catalytic hydrogenation in competitive reactions, we have found that splitting of the substituted benzyl ethers such as PMB did not start until the removal of the extended aromatic system became complete. $^{12,13}$

To explore the utility of this observation, a series of mono- and disaccharides carrying NAP and PMB groups were prepared and subjected to catalytic hydrogenation.

\section{Results and Discussion}

Fully protected glucoside derivatives $\mathbf{2}$ and $\mathbf{5}$ carrying benzyl, p-methoxybenzyl and 2(naphthyl)methyl protecting groups were prepared by conventional alkylation, starting from $\mathbf{1}^{14}$ and $4,{ }^{14}$ respectively. Tlc monitoring of the $\mathrm{Pd} / \mathrm{C}$-catalyzed hydrogenolysis of $\mathbf{2}$ showed formation of a single product until the conversion reached at $80-90 \%$; prolongation of the reaction time led to the formation of some polar side products. Therefore, the reaction mixture was worked up at $90 \%$ completion, and compound $\mathbf{3}$, selectively deprotected at position 4, could be isolated with $68 \%$ yield. Similarly, the NAP group could be removed from primary hydroxyl group of 5 in the presence of PMB and benzyl ethers, to yield $\mathbf{6}$ (Scheme 1).

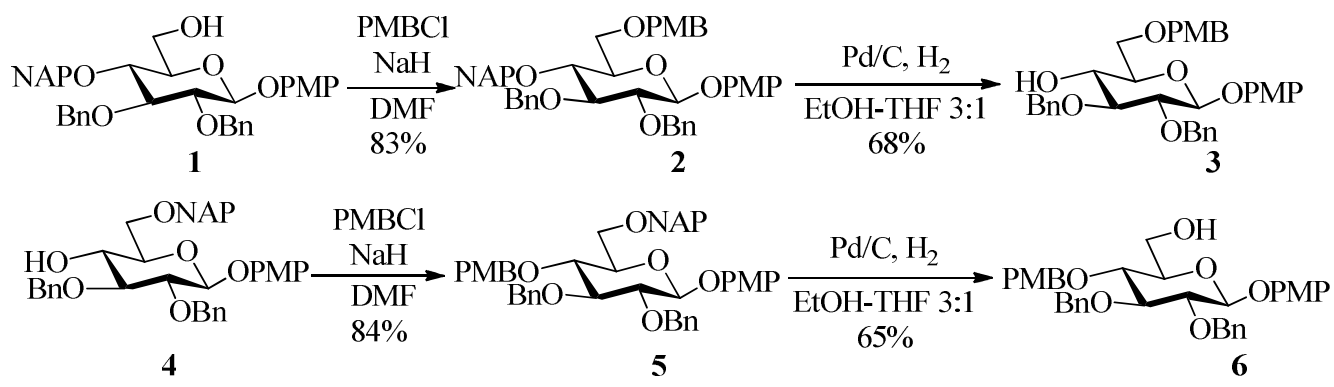

Scheme 1. Removal of the (2-naphthyl)methyl group in the presence of the $p$-methoxybenzyl PMB and benzyl groups by catalytic hydrogenation.

Compound 7 was alkylated with (2-naphthyl)methyl bromide in the presence of phase transfer catalyst $\left(\mathrm{Bu}_{4} \mathrm{NHSO}_{4}\right)$ affording a mixture of $\mathbf{8}^{15}$ and $\mathbf{9}$, respectively, whose separation has been accomplished by column chromatography. Subsequently, their $p$-methyoxybenzylation gave the acetal-containing substrates $\mathbf{1 0}$ and 12. Catalytic hydrogenolysis of $\mathbf{1 0}$ was let to go to 
completion, furnishing the mono-deprotected 11. ${ }^{16}$ The NAP group of $\mathbf{1 2}$ could be selectively cleaved, as well, to give $\mathbf{1 3}^{16}$ in high yield (Scheme 2).

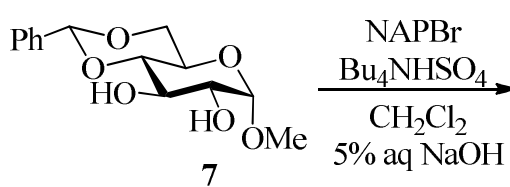

7
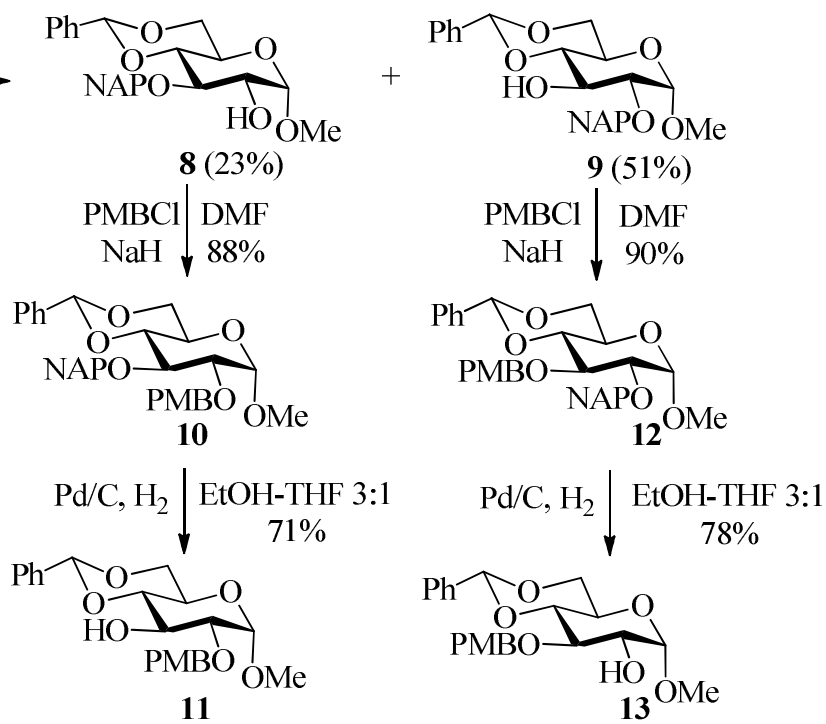

Scheme 2. Removal of the (2-naphthyl)methyl group in the presence of the $p$-methoxybenzyl and benzylidene groups.

Starting from the galactopyranoside derivative 14, the synthesis of $\mathbf{1 7}$ and $\mathbf{1 8}$ was achieved by an analogous manner $(\mathbf{1 4} \rightarrow \mathbf{1 5} \rightarrow \mathbf{1 7} ; \mathbf{1 4} \rightarrow \mathbf{1 6} \rightarrow \mathbf{1 8})$, but the regioisomers formed in the phase transfer alkylation could only be separated after the introduction of the PMB group. The NAP group of $\mathbf{1 7}$ and $\mathbf{1 8}$ could be cleanly removed in the presence of the PMB and benzylidene functions to result in $\mathbf{1 9}$ and $\mathbf{2 0}$ in high yields, respectively. Compounds 17-20 were identified after the synthesis and structural elucidation of the acetylated derivative $\mathbf{2 1}$ (Scheme 3). 


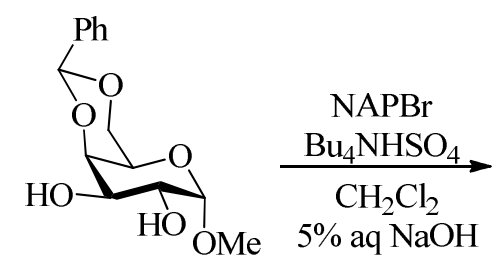

14

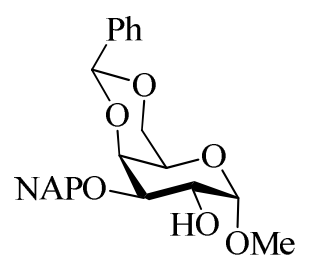

15

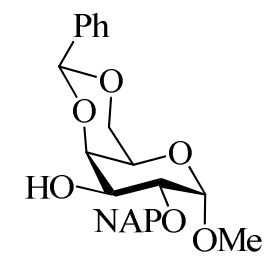

16

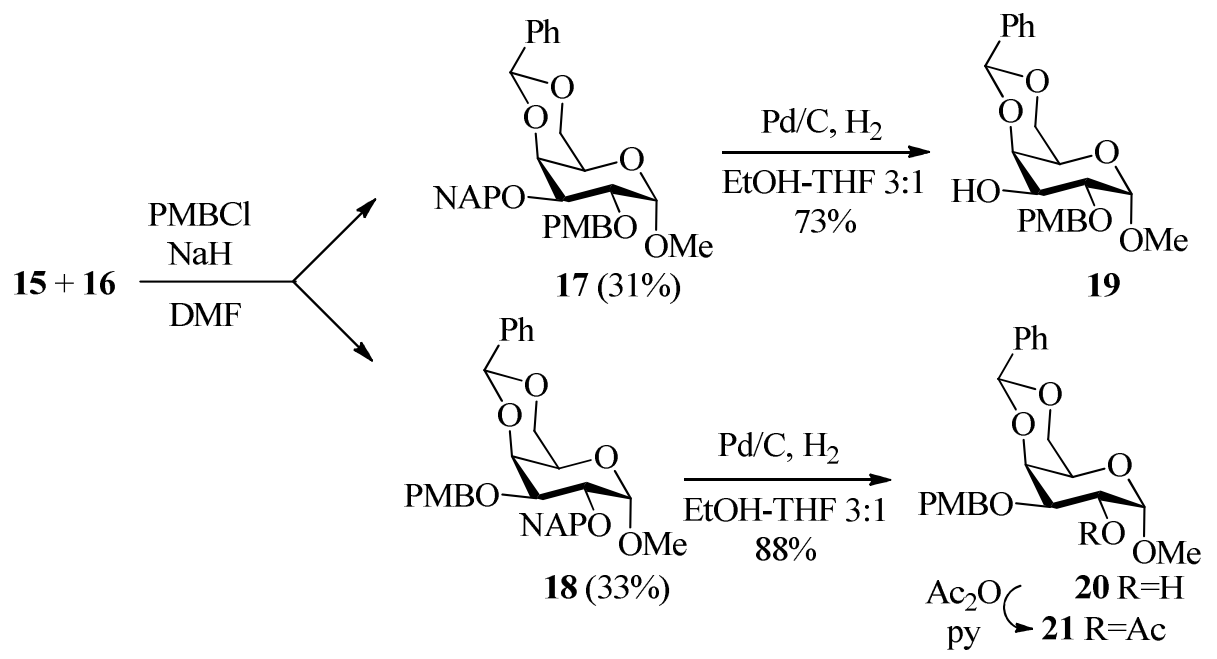

Scheme 3. Removal of the (2-naphthyl)methyl group in the presence of the $p$-methoxybenzyl and benzylidene groups.

Orthogonal deprotection of the NAP group was studied at a disaccharide level, too. 2(Napthyl)methylation of the single free hydroxyl group of the 3,4-O-Isopropylidene-6-O(methoxydimethyl)methyl- $\beta$-D-galactopyranosyl-( $1 \rightarrow 6)$-1,2:3,4-di- $O$-isopropylidene- $\alpha$-Dgalactopyranose $\mathbf{2 2}{ }^{17}$ afforded $\mathbf{2 3}$ from which, after mild acidic hydrolysis of the mixed acetal group followed by $p$-methoxybenzylation compound 25 could be obtained. Upon catalytic hydrogenation, the NAP group could be cleaved selectively form the secondary hydroxyl group to give $\mathbf{2 6}$ in high yield (Scheme 4). 

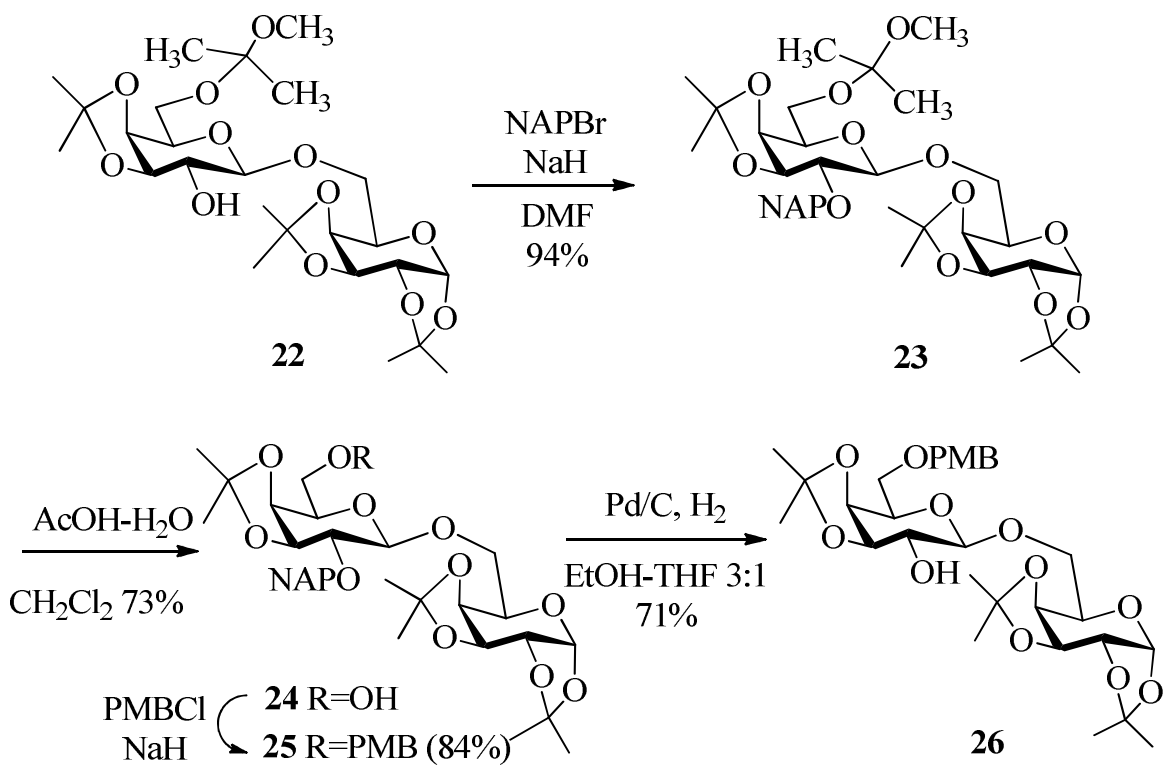

Scheme 4. Removal of the (2-naphthyl)methyl group in the presence of isopropylidene groups by catalytic hydrogenation.

Treatment of the known lactoside $27^{18}$ with $p$-methoxybenzyl chloride as a reagent furnished $\mathbf{2 8}$, as another disaccharide substrate, suitable for studying the selective removal of NAP in the presence of PMB and multiple benzyl ethers. Catalytic hydrogenolysis of $\mathbf{2 8}$ clearly demonstrated the limitation of the method, since the monodeproteced $\mathbf{2 9}$ could be isolated in rather low yield (Scheme 5). A side product formed in this transformation was also isolated in a similar amount; it could be identified, by NMR measurments and its single peak in the mass spectrum (m/z: 951.30), as a mixture of mono-debenzylated derivatives of 29 ( $\mathrm{m} / \mathrm{z}$ calcd for $[\mathrm{M}+\mathrm{Na}]^{+}:$951.40).

The higher hydrogenolytic stability of the $p$-methoxybenzyl ether in comparison to the benzyl can be explained by steric effect of the methoxy substituent which inhibits the effective binding of the aromatic ring to the catalyst surface. ${ }^{6}$ This phenomenon was utilized for selective hydrogenation of phenolic benzyl ether or ester in the presence of phenolic PMB ether using a $\mathrm{Pd} / \mathrm{C}$-pyridine combination as a catalyst. ${ }^{19,20}$ 

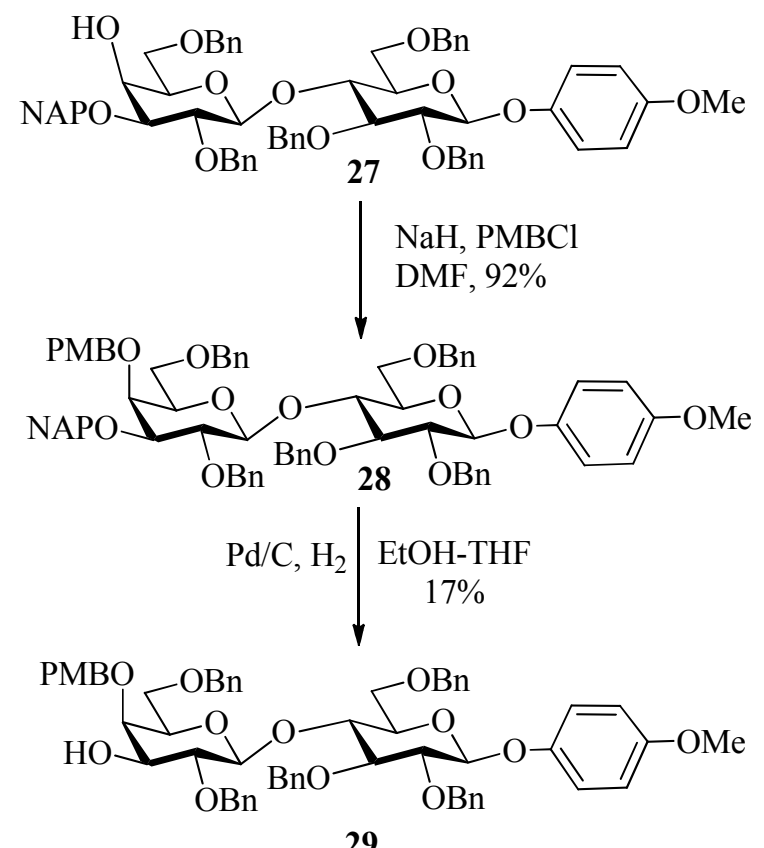

Scheme 5. Removal of the (2-naphthyl)methyl group in the presence of benzyl groups by catalytic hydrogenation.

\section{Conclusions}

The (2-naphthyl)methyl (NAP) protecting group could be selectively removed by catalytic hydrogenation from primary and secondary hydroxyl groups in the presence of the $p$ methoxybenzyl (PMB), benzylidene and multiple benzyl functions in the case of monosaccharides. At a disaccharide level, the $p$-methoxybenzyl (PMB) group proved to be stable toward catalytic hydroganolysis, however, partial cleavage of benzyl ether functions was also observed.

\section{Experimental Section}

General. Optical rotations were measured at room temperature with a Perkin-Elmer 241 automatic polarimeter. TLC was performed on Kieselgel $60 \mathrm{~F}_{254}$ (Merck) with detection by immersing into $5 \%$ ethanolic sulfuric acid soln followed by heating. Column chromatography was performed on Silica gel 60 (Merck 0.063-0.200 mm). Organic solutions were dried over $\mathrm{MgSO}_{4}$, and concentrated in vacuum. The ${ }^{1} \mathrm{H}(360,400$ and $500 \mathrm{MHz})$ and ${ }^{13} \mathrm{C} \mathrm{NMR}(90.54$, 100.28 and 125.76 MHz) spectra were recorded with Bruker DRX-360, Bruker DRX-400 and Bruker DRX-500 spectrometers. Chemical shifts are referenced to $\mathrm{Me}_{4} \mathrm{Si}\left(0.00 \mathrm{ppm}\right.$ for $\left.{ }^{1} \mathrm{H}\right)$ or to 
the residual solvent signals $\left(\mathrm{CDCl}_{3}\right.$ : $77.00 \mathrm{ppm}$ for $\left.{ }^{13} \mathrm{C}\right)$. MALDI-TOF MS analyses of the compounds were carried out in the positive reflectron mode using a BIFLEX III mass spectrometer (Bruker, Germany) equipped with delayed-ion extraction. The matrix solution was a satd 2,4,6-trihydroxy-acetophenone (THAP) solution in MeCN. A Vario MICRO CUBE instrument was used for elemental analyses.

p-Methoxyphenyl 2,3-di- $O$-benzyl-6- $O$-( $p$-methoxybenzyl)-4- $O$-(2-naphthyl)methyl- $\beta$-Dglucopyranoside (2). Compound $\mathbf{1}^{14}$ (694 mg, $1.14 \mathrm{mmol}$ ) was dissolved in DMF (10 ml) then $60 \% \mathrm{NaH}\left(69 \mathrm{mg}, 1.5\right.$ equiv) was added at $0^{\circ} \mathrm{C}$ and it was stirred for $30 \mathrm{~min}$. $p$-Methoxybenzyl chloride $(0.15 \mathrm{~mL}, 1.3$ equiv) was added to the reaction mixture and stirred for further 15 min at $0^{\circ} \mathrm{C}$ and $1 \mathrm{~h}$ at room temperature. The mixture was evaporated, diluted with DCM, washed with water $(4 \mathrm{x})$, dried, filtered and evaporated. The crude product was purified by column chromatography (DCM/acetone 95:5) to yield 2 as white needles $(654 \mathrm{mg}, 83 \%) . \mathrm{mp}=83-85^{\circ} \mathrm{C}$; $[\alpha]_{\mathrm{D}}{ }^{25}-8.51\left(c 0.67, \mathrm{CHCl}_{3}\right) .{ }^{1} \mathrm{H} \mathrm{NMR}\left(360 \mathrm{MHz}, \mathrm{CDCl}_{3}\right): \delta_{\mathrm{H}} 7.81-6.68(25 \mathrm{H}, \mathrm{m}$, arom.), 5.07$4.44\left(9 \mathrm{H}, \mathrm{m}, 4 \times \mathrm{CH}_{2}, \mathrm{H}-1\right), 3.81-3.67(5 \mathrm{H}, \mathrm{m}), 3.75\left(3 \mathrm{H}, \mathrm{s}, \mathrm{OCH}_{3}\right), 3.71\left(3 \mathrm{H}, \mathrm{s}, \mathrm{OCH}_{3}\right), 3.59-$ $3.58(1 \mathrm{H}, \mathrm{m}) .{ }^{13} \mathrm{C}$ NMR (90 MHz, $\mathrm{CDCl}_{3}$ ): $\delta_{\mathrm{C}} 159.1-113.6$ (arom.), 102.7 (C-1), 84.6, 82.0, 77.6 (C-2, C-3, C-4, C-5), 75.7, 74.9, $73.1\left(4 \mathrm{x} \mathrm{CH}_{2}\right), 68.4$ (C-6), 55.5, 55.1 (2 x $\left.\mathrm{OCH}_{3}\right)$. Anal. Calcd for $\mathrm{C}_{46} \mathrm{H}_{46} \mathrm{O}_{8}$ (726.85): C, 76.01; H,6.38. Found: C, 76.24; H,6. 36.

$\boldsymbol{p}$-Methoxyphenyl 2,3-di- $\boldsymbol{O}$-benzyl-6- $\boldsymbol{O}$-( $\boldsymbol{p}$-methoxybenzyl)- $\boldsymbol{\beta}$-D-glucopyranoside (3). To a solution of compound 2 (218 mg, $0.03 \mathrm{~mol})$ in a mixture of EtOH and THF $(3: 1,20 \mathrm{~mL}) \mathrm{Pd}$ on carbon $(30 \mathrm{mg}$ ) was added and the mixture was stirred in hydrogen atmosphere until TLC showed $90 \%$ conversion (6h). It was then filtered through a layer of Celite and concentrated. The crude product was purified by column chromatography (DCM/EtOAc 96:4) to yield compound 3 as white needles $(120 \mathrm{mg}, 68 \%) . \mathrm{mp}=73-74{ }^{\circ} \mathrm{C} ;[\alpha]_{\mathrm{D}}{ }^{25}-25.65\left(c 0.46, \mathrm{CHCl}_{3}\right) .{ }^{1} \mathrm{H} \mathrm{NMR}(360$ $\left.\mathrm{MHz}_{\mathrm{CDCl}}\right): \delta_{\mathrm{H}} 7.32-6.79\left(18 \mathrm{H}, \mathrm{m}\right.$, arom.), 5.06-4.74 (5H, m, $\left.2 \times \mathrm{CH}_{2}, \mathrm{H}-1\right), 4.47,4.51(2 \mathrm{H}$, $\left.2 \mathrm{~d}, J_{\text {gem }}=10.5 \mathrm{~Hz}, \mathrm{CH}_{2}\right), 3.78\left(3 \mathrm{H}, \mathrm{s}, \mathrm{OCH}_{3}\right), 3.76\left(4 \mathrm{H}, \mathrm{s}, \mathrm{OCH}_{3}\right.$, skeleton $\left.\mathrm{H}\right), 3.70-3.62(3 \mathrm{H}$, $\mathrm{m}), 3.54-3.49(2 \mathrm{H}, \mathrm{m}), 2.65(1 \mathrm{H}, \mathrm{s}, \mathrm{OH}) .{ }^{13} \mathrm{C} \mathrm{NMR}\left(90 \mathrm{MHz}, \mathrm{CDCl}_{3}\right): \delta_{\mathrm{C}} 159.2-113.7$ (arom.), 102.7 (C-1), 83.9, 81.4, 74.2, 71.4 (C-2, C-3, C-4, C-5), 75.3, 74.8, $73.2\left(3 \times \mathrm{CH}_{2}\right), 69.8$ (C-6), 55.5, $55.2\left(2 \mathrm{x} \mathrm{OCH}_{3}\right)$. Anal. Calcd for $\mathrm{C}_{35} \mathrm{H}_{38} \mathrm{O}_{8}$ (586.26): C, 71.65; H, 6.53. Found: C, 71.50; $\mathrm{H}, 6.55$. MALDI-TOF m/z calcd for $[\mathrm{M}+\mathrm{Na}]^{+}: 609.66$, Found: 609.41.

p-Methoxyphenyl 2,3-di- $O$-benzyl-4- $O$-( $\boldsymbol{p}$-methoxybenzyl)-6-O-(2-naphthyl)methyl- $\beta$-Dglucopyranoside (5). Compound $4^{14}(519 \mathrm{~g}, 0.86 \mathrm{mmol})$ was treated with $p$-methoxybenzyl chloride $(113 \mu \mathrm{L}, 1.2$ equiv) as described for the synthesis of compound $\mathbf{2}$ to yield compound $\mathbf{5}$ as a white powder (533 mg, 84\%). (Column chromatography: DCM/acetone 95:5). $\mathrm{mp}=91-93$ ${ }^{\circ} \mathrm{C} ;[\alpha]_{\mathrm{D}}{ }^{25}-8.3\left(c 0.53, \mathrm{CHCl}_{3}\right) .{ }^{1} \mathrm{H}$ NMR $\left(360 \mathrm{MHz}, \mathrm{CDCl}_{3}\right): \delta_{\mathrm{H}} 7.76-6.70(25 \mathrm{H}, \mathrm{m}$, arom.), 5.04-4.71 (9H, m, 4 x CH $2, \mathrm{H}-1), 3.83-3.59(6 \mathrm{H}, \mathrm{m}), 3.72\left(3 \mathrm{H}, \mathrm{s}, \mathrm{OCH}_{3}\right), 3.70\left(3 \mathrm{H}, \mathrm{s}, \mathrm{OCH}_{3}\right)$. ${ }^{13} \mathrm{C}$ NMR $\left(90 \mathrm{MHz}, \mathrm{CDCl}_{3}\right): \delta_{\mathrm{C}} 159.2-113.6$ (arom.), $102.6(\mathrm{C}-1), 84.6,82.0,77.3,75.0(\mathrm{C}-2$, C3, C-4, C-5), 75.7, 74.9, 74.6, 73.4 (4 x CH$), 68.7$ (C-6), 55.5, 55.1 (2 x $\left.\mathrm{OCH}_{3}\right)$. Anal. Calcd for $\mathrm{C}_{46} \mathrm{H}_{46} \mathrm{O}_{8}$ (726.85): C, 76.01; H, 6.38. Found: C, 75.78; H, 6.41. 
p-Methoxyphenyl 2,3-di- $\boldsymbol{O}$-benzyl-4- $\boldsymbol{O}$-( $\boldsymbol{p}$-methoxybenzyl)- $\boldsymbol{\beta}$-D-glucopyranoside

(6).

Compound 5 (218 mg, $1.05 \mathrm{mmol})$ was hydrogenolysed as described for the synthesis of compound 3 to obtain 6 as white powder (132 mg, 65\%). (Column chromatography: DCM/EtOAc 96:4). mp = 110-111 ${ }^{\circ} \mathrm{C} ;[\alpha]_{\mathrm{D}}{ }^{25}-10.71\left(c \quad 0.42, \mathrm{CHCl}_{3}\right) .{ }^{1} \mathrm{H}$ NMR $(360 \mathrm{MHz}$, $\left.\mathrm{CDCl}_{3}\right): \delta_{\mathrm{H}} 7.33-6.81(18 \mathrm{H}, \mathrm{m}$, arom.), 5.03-4.57 (7H, m, 3 x CH $2, \mathrm{H}-1), 3.88-3.84(1 \mathrm{H}, \mathrm{m}), 3.77$ $\left(3 \mathrm{H}, \mathrm{s}, \mathrm{OCH}_{3}\right), 3.76\left(3 \mathrm{H}, \mathrm{s}, \mathrm{OCH}_{3}\right), 3.72-3.41(5 \mathrm{H}, \mathrm{m}), 2.15(1 \mathrm{H}, \mathrm{s}, \mathrm{OH}) .{ }^{13} \mathrm{C} \mathrm{NMR}(90 \mathrm{MHz}$, $\mathrm{CDCl}_{3}$ ): $\delta_{\mathrm{C}}$ 159.3-113.8 (arom.), 102.4 (C-1), 84.4, 82.0, 77.1, 75.2 (C-2, C-3, C-4, C-5), 75.6, 75.0, $74.7\left(3 \times \mathrm{CH}_{2}\right), 61.9$ (C-6), 55.6, $55.2\left(2 \times \mathrm{OCH}_{3}\right)$. Anal. Calcd for $\mathrm{C}_{35} \mathrm{H}_{38} \mathrm{O}_{8}$ (586.26): $\mathrm{C}$, 71.65; H, 6.53. Found: C, 71.82; H, 6.57. MALDI-TOF m/z calcd for $[\mathrm{M}+\mathrm{Na}]^{+}: 609.66$, Found: 609.43 .

Methyl 4,6-O-benzylidene-3- $O$-(2-naphthyl)methyl- $\alpha$-D-glucopyranoside (8) and methyl 4,6$\boldsymbol{O}$-benzylidene-2-O -(2-naphthyl)methyl- $\alpha$-D-glucopyranoside (9). To a stirred solution of 7 (19.00 g, $67 \mathrm{mmol})$ in DCM (500 mL) $\mathrm{Bu}_{4} \mathrm{NHSO}_{4}(2.8 \mathrm{~g}, 8.2 \mathrm{mmol}), 5 \%$ aq. $\mathrm{NaOH}(200 \mathrm{~mL})$ and 2-(bromomethyl)naphthalene (20.8 g, $94 \mathrm{mmol}, 1.4$ eqiuv) were added, then it was stirred for 24 hours at reflux temperature. The organic layer was then separated, diluted with DCM, washed with water, dried, filtered and concentrated. The crude product was purified by column chromatography ( $n$-hexane/EtOAc 6:4) to yield compound $\mathbf{8}^{15}$ and $\mathbf{9}$.

(8) White needles, yield: $6.55 \mathrm{~g}(23 \%)$, mp. $199-201^{\circ} \mathrm{C},[\alpha]_{\mathrm{D}}{ }^{25}+71.73\left(c 0.19, \mathrm{CHCl}_{3}\right)$, (lit. 15 $\left.[\alpha]_{\mathrm{D}}{ }^{25}+69.9\right) ; \mathrm{R}_{f} 0.28$ (n-hexane/EtOAc 6:4). ${ }^{1} \mathrm{H}$ NMR $\left(500 \mathrm{MHz}, \mathrm{CDCl}_{3}\right): \delta_{\mathrm{H}} 7.78-7.35(12 \mathrm{H}$, $\mathrm{m}$, arom.), $5.52\left(1 \mathrm{H}, \mathrm{s}, \mathrm{H}_{\mathrm{ac}}\right), 5.07-4.92\left(2 \mathrm{H}, 2 \mathrm{~d}, \mathrm{NAP}-\mathrm{CH}_{2}\right), 4.73\left(1 \mathrm{H}, \mathrm{d},{ }^{3} J_{1,2}=4.0 \mathrm{~Hz}, \mathrm{H}-1\right)$, 4.27-4.24 (1H, m), $3.85(1 \mathrm{H}, \mathrm{t}, J=9.5 \mathrm{~Hz}), 3.81-3.78(1 \mathrm{H}, \mathrm{m}), 3.72-3.70(2 \mathrm{H}, \mathrm{m}), 3.60(1 \mathrm{H}, \mathrm{t}, J$ $=9.5 \mathrm{~Hz}), 3.38\left(3 \mathrm{H}, \mathrm{s}, \mathrm{OCH}_{3}\right), 2.26(1 \mathrm{H}, \mathrm{d}, J=8.2 \mathrm{~Hz}, \mathrm{OH}) .{ }^{13} \mathrm{C} \mathrm{NMR}\left(125.7 \mathrm{MHz}, \mathrm{CDCl}_{3}\right): \delta_{\mathrm{C}}$ 138.37-125.0 (arom.), $101.2\left(\mathrm{C}_{\mathrm{ac}}\right), 99.9$ (C-1), 81.7, 78.7, 72.1, 62.4 (C-2, C-3, C-4, C-5), 74.6 (NAP-CH$), 68.8(\mathrm{C}-6), 55.2\left(\mathrm{OCH}_{3}\right)$. Anal. Calcd for $\mathrm{C}_{25} \mathrm{H}_{26} \mathrm{O}_{6}(422.47)$ : C, 71.07; H, 6.20. Found: C, 71.29; H, 6.17.

(9) White needles, yield: $14.49 \mathrm{~g}(51 \%)$. $[\alpha]_{\mathrm{D}}{ }^{25}+9.85\left(c 0.13, \mathrm{CHCl}_{3}\right), \mathrm{mp}=165-166{ }^{\circ} \mathrm{C}, \mathrm{R}_{f} 0.43$ (n-hexane/EtOAc 6:4). ${ }^{1} \mathrm{H}$ NMR (500 MHz, $\left.\mathrm{CDCl}_{3}\right)$ : $\delta_{\mathrm{H}} 7.77-7.29(12 \mathrm{H}, \mathrm{m}$, arom.), $5.46(1 \mathrm{H}, \mathrm{s}$, $\left.\mathrm{H}_{\mathrm{ac}}\right)$, 4.91-4.78 (2H, 2d, NAP-CH $\mathrm{CH}_{2}, 4.57\left(1 \mathrm{H}, \mathrm{d},{ }^{3} J_{1,2}=3.5 \mathrm{~Hz}, \mathrm{H}-1\right), 4.22-4.15(2 \mathrm{H}, \mathrm{m}), 3.82-$ $3.75(1 \mathrm{H}, \mathrm{m}), 3.62(1 \mathrm{H}, \mathrm{t}, J=10.5 \mathrm{~Hz}), 3.48-3.43(2 \mathrm{H}, \mathrm{m}), 3.32\left(3 \mathrm{H}, \mathrm{s}, \mathrm{OCH}_{3}\right), 2.88(1 \mathrm{H}, \mathrm{d}, J=$ $2.2 \mathrm{~Hz}, \mathrm{OH}) .{ }^{13} \mathrm{C}$ NMR $\left(125.7 \mathrm{MHz}, \mathrm{CDCl}_{3}\right): \delta_{\mathrm{C}} 137.0-125.7$ (arom.), $101.1\left(\mathrm{C}_{\mathrm{ac}}\right), 98.5(\mathrm{C}-1)$, 81.1, 79.3, 70.1, 61.9 (C-2, C-3, C-4, C-5), $73.3\left(\mathrm{NAP}_{-} \mathrm{CH}_{2}\right), 68.8(\mathrm{C}-6), 55.2\left(\mathrm{OCH}_{3}\right)$. Anal. Calcd for $\mathrm{C}_{25} \mathrm{H}_{26} \mathrm{O}_{6}$ (422.47): C, 71.07; H, 6.20. Found: C, 71.14; H, 6.22. MALDI-TOF m/z calcd for $[\mathrm{M}+\mathrm{Na}]^{+}: 445.46$, Found: 445.21 .

Methyl 4,6- $O$-benzylidene-2- $O$-(p-methoxybenzyl)-3-O-(2-naphthyl)methyl- $\alpha$-Dglucopyranoside (10). Compound $8(1.78 \mathrm{~g}, 9.59 \mathrm{mmol})$ was treated with $p$-methoxybenzyl chloride ( $0.69 \mathrm{~mL}, 1.2$ equiv) as described for the synthesis of compound 2 to yield compound 10 as white crystals $(2.37 \mathrm{~g}, 88 \%$ ). (Column chromatography: DCM/EtOAc 97:3) mp = 94-95 ${ }^{\circ} \mathrm{C},[\alpha]_{\mathrm{D}}{ }^{25}+45.04,\left(c 0.13, \mathrm{CHCl}_{3}\right) .{ }^{1} \mathrm{H}$ NMR $\left(500 \mathrm{MHz}, \mathrm{CDCl}_{3}\right): \delta_{\mathrm{H}} 7.80-6.81(16 \mathrm{H}, \mathrm{m}$, arom.), $5.54\left(1 \mathrm{H}, \mathrm{s}, \mathrm{H}_{\mathrm{ac}}\right), 5.06-4.96\left(2 \mathrm{H}, 2 \mathrm{~d}, \mathrm{NAP}-\mathrm{CH}_{2}\right), 4.78-4.63\left(2 \mathrm{H}, \mathrm{dd}, \mathrm{PMB}-\mathrm{CH}_{2}\right), 4.56\left(1 \mathrm{H}, \mathrm{d},{ }^{3} J_{1,2}\right.$ $=3.5 \mathrm{~Hz}, \mathrm{H}-1), 4.26-4.23(1 \mathrm{H}, \mathrm{m}), 4.07(1 \mathrm{H}, \mathrm{t}, J=9.0 \mathrm{~Hz}), 3.85-3.79(1 \mathrm{H}, \mathrm{m}), 3.73(3 \mathrm{H}, \mathrm{s}$, 
PMB-OCH $)_{3}, 3.69(1 \mathrm{H}, \mathrm{t}, J=10.0 \mathrm{~Hz}), 3.61(1 \mathrm{H}, \mathrm{t}, J=9.0 \mathrm{~Hz}), 3.56-3.54(1 \mathrm{H}, \mathrm{m}), 3.37(3 \mathrm{H}, \mathrm{s}$, $\left.\mathrm{OCH}_{3}\right) .{ }^{13} \mathrm{C} \mathrm{NMR}\left(125.7 \mathrm{MHz}, \mathrm{CDCl}_{3}\right): \delta_{\mathrm{C}} 159.3-113.8$ (arom.), $101.9\left(\mathrm{C}_{\mathrm{ac}}\right), 99.2(\mathrm{C}-1), 82.1$, 78.9, 78.5, 62.3 (C-2, C-3, C-4, C-5), 75.2, 73.2 (2 x CH$), 69.0$ (C-6), 55.2, 55.1 (2 x $\left.\mathrm{OCH}_{3}\right)$. Anal. Calcd for $\mathrm{C}_{33} \mathrm{H}_{34} \mathrm{O}_{7}$ (542.62): C, 73.04; H, 6.32. Found: C, 73.22; H, 6.35.

Methyl 4,6-O-benzylidene-2- $O$-( $\boldsymbol{p}$-methoxybenzyl)- $\alpha$-D-glucopyranoside (11). To a solution of compound $10(160 \mathrm{mg}, 0.23 \mathrm{mmol})$ in a mixture of EtOH and THF (3:1, $20 \mathrm{~mL}) \mathrm{Pd}$ on carbon (30 mg) was added and the mixture was stirred in hydrogen atmosphere until TLC showed complete conversion (6h). It was then filtered through a layer of Celite and evaporated. The crude product was purified by column chromatography (DCM/acetone 96:4) to yield compound 11 as white crystals $(84 \mathrm{mg}, 71 \%) . \mathrm{mp}=98-100{ }^{\circ} \mathrm{C},[\alpha]_{\mathrm{D}}{ }^{25}+78.71\left(c 0.16, \mathrm{CHCl}_{3}\right)$, (lit. 16: $\mathrm{mp}=$ $\left.102{ }^{\circ} \mathrm{C},[\alpha]_{\mathrm{D}}+38\right) .{ }^{1} \mathrm{H}$ NMR $\left(360 \mathrm{MHz}, \mathrm{CDCl}_{3}\right): \delta_{\mathrm{H}} 7.50-6.87(9 \mathrm{H}, \mathrm{m}$, arom. $), 5.51\left(1 \mathrm{H}, \mathrm{s}, \mathrm{H}_{\mathrm{ac}}\right)$, 4.72-4.61 (2H, dd, PMB-CH $), 4.56\left(1 \mathrm{H}, \mathrm{d},{ }^{3} J_{1,2}=3.6 \mathrm{~Hz}, \mathrm{H}-1\right), 4.27-4.23(1 \mathrm{H}, \mathrm{m}), 4.12(1 \mathrm{H}, \mathrm{t}, J$ $=9.3 \mathrm{~Hz}), 3.83-3.76(1 \mathrm{H}, \mathrm{m}), 3.79\left(3 \mathrm{H}, \mathrm{s}, \mathrm{PMB}-\mathrm{OCH}_{3}\right), 3.69(1 \mathrm{H}, \mathrm{t}, J=10.1 \mathrm{~Hz}), 3.51-3.42(2 \mathrm{H}$, m), $3.36\left(3 \mathrm{H}, \mathrm{s}, \mathrm{OCH}_{3}\right), 2.72(1 \mathrm{H}, \mathrm{bs}, \mathrm{OH}) .{ }^{13} \mathrm{C} \mathrm{NMR}\left(90 \mathrm{MHz}, \mathrm{CDCl}_{3}\right): \delta_{\mathrm{C}} 159.5-113.9$ (arom.),

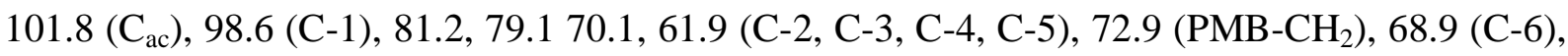
55.3, $55.2\left(2 \times \mathrm{OCH}_{3}\right)$. Anal. Calcd for $\mathrm{C}_{22} \mathrm{H}_{26} \mathrm{O}_{7}$ (402.44): C, 65.66; H, 6.51; O. Found: C, $65.54 ; \mathrm{H}, 6.49$.

Methyl 4,6-O-benzylidene-3- $O$-(p-methoxybenzyl)-2- $O$-(2-naphthyl)methyl- $\alpha$-Dglucopyranoside (12). Compound $9(4.05 \mathrm{~g}, 9.56 \mathrm{mmol})$ was treated with $p$-methoxybenzyl chloride as described for the synthesis of compound $\mathbf{2}$ to give $\mathbf{1 2}$ as white crystals $(4.56 \mathrm{~g}, 90 \%)$. $\mathrm{mp}=96-98^{\circ} \mathrm{C},[\alpha]_{\mathrm{D}}{ }^{25}-49.19\left(c 0.62, \mathrm{CHCl}_{3}\right) .{ }^{1} \mathrm{H}$ NMR $\left(500 \mathrm{MHz}, \mathrm{CDCl}_{3}\right): \delta_{\mathrm{H}} 7.81-6.80(16 \mathrm{H}$,

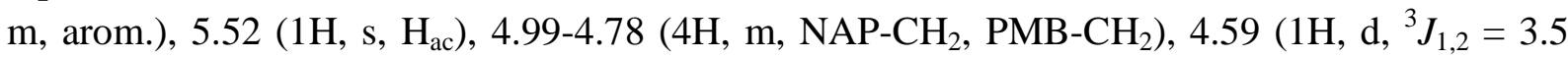
$\mathrm{Hz}, \mathrm{H}-1), 4.25-4.22(1 \mathrm{H}, \mathrm{m}), 4.06(1 \mathrm{H}, \mathrm{t}, J=9.5 \mathrm{~Hz}), 3.84-3.78(1 \mathrm{H}, \mathrm{m}), 3.74(3 \mathrm{H}, \mathrm{s}$, PMB$\left.\mathrm{OCH}_{3}\right), 3.66(1 \mathrm{H}, \mathrm{t}, J=10.0 \mathrm{~Hz}), 3.59-3.55(2 \mathrm{H}, \mathrm{m}), 3.39\left(3 \mathrm{H}, \mathrm{s}, \mathrm{OCH}_{3}\right) .{ }^{13} \mathrm{C} \mathrm{NMR}(125.7 \mathrm{MHz}$, $\mathrm{CDCl}_{3}$ ): $\delta_{\mathrm{C}} 159.1-113.6$ (arom.), $101.1\left(\mathrm{C}_{\mathrm{ac}}\right), 99.1(\mathrm{C}-1), 82.1,78.9,78.5,62.3$ (C-2, C-3, C-4, C5), 74.2, $73.7\left(2 \times \mathrm{CH}_{2}\right), 68.9(\mathrm{C}-6), 55.2,55.1\left(2 \times \mathrm{OCH}_{3}\right)$. Anal. Calcd for $\mathrm{C}_{33} \mathrm{H}_{34} \mathrm{O}_{7}(542.62)$ : C, 73.04; H, 6.32. Found: C, 72.89; H, 6.30.

Methyl 4,6-O-benzylidene-3-O-(p-methoxybenzyl)-a-D-glucopyranoside (13). Compound 12 (160 mg, $0.23 \mathrm{mmol}$ ) was hydrogenolysed as described for the synthesis of compound 11 to obtain 13 as white crystals $(92 \mathrm{mg}, 78 \%) . \mathrm{mp}=168-169^{\circ} \mathrm{C},[\alpha]_{\mathrm{D}}{ }^{25}+78.53\left(c 0.11, \mathrm{CHCl}_{3}\right)$, (lit. 16: $\left.\mathrm{mp}=174-175{ }^{\circ} \mathrm{C},[\alpha]_{\mathrm{D}}+45\right) .{ }^{1} \mathrm{H} \mathrm{NMR}\left(360 \mathrm{MHz}, \mathrm{CDCl}_{3}\right): \delta_{\mathrm{H}} 7.51-6.83(9 \mathrm{H}, \mathrm{m}$, arom. $), 5.56$ $\left(1 \mathrm{H}, \mathrm{s}, \mathrm{H}_{\mathrm{ac}}\right), 4.89-4.70\left(2 \mathrm{H}, 2 \mathrm{~d}, \mathrm{PMB}-\mathrm{CH}_{2}\right), 4.78\left(1 \mathrm{H}, \mathrm{d},{ }^{3} J_{1,2}=3.8 \mathrm{~Hz}, \mathrm{H}-1\right), 4.31-4.27(1 \mathrm{H}, \mathrm{m})$, 3.86-3.64 (4H, m), $3.78\left(3 \mathrm{H}, \mathrm{s}, \mathrm{PMB}-\mathrm{OCH}_{3}\right), 3.62(1 \mathrm{H}, \mathrm{t}, J=9.1 \mathrm{~Hz}), 3.44\left(3 \mathrm{H}, \mathrm{s}, \mathrm{OCH}_{3}\right), 2.39$ $(1 \mathrm{H}, \mathrm{d}, J=7.2 \mathrm{~Hz}, \mathrm{OH}) .{ }^{13} \mathrm{C} \mathrm{NMR}\left(90 \mathrm{MHz}, \mathrm{CDCl}_{3}\right): \delta_{\mathrm{C}} 159.2-113.7$ (arom.), $101.2\left(\mathrm{C}_{\mathrm{ac}}\right), 99.8$

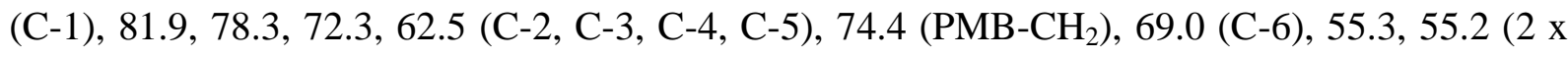
$\mathrm{OCH}_{3}$ ). Anal. Calcd for $\mathrm{C}_{22} \mathrm{H}_{26} \mathrm{O}_{7}$ (402.44): C, 65.66; H, 6.51. Found: $\mathrm{C}, 65.71 ; \mathrm{H}, 6.55$.

Methyl 4,6-O-benzylidene-2- $O$-( $p$-methoxybenzyl)-3- $O$-(2-naphthyl)methyl- $\alpha$-Dgalactopyranoside (17) and methyl 4,6-O-benzylidene-3- $O$ - $(p$-methoxybenzyl)-2- $O$-(2naphthyl)methyl-a-D-galactopyranoside (18). Compound $14(840 \mathrm{mg}, 3 \mathrm{mmol})$ was treated with 2-(bromomethyl)naphthalene as described for the synthesis of compound $\mathbf{8}$ and $\mathbf{9}$ to give the 
unseparated, purified mixture of compound 15 and 16 (993 mg, 79\%). (Column chromatography: toluene: acetone $75: 25, \mathrm{R}_{f} 0.40$ and 0.35 .)

Purified mixture of compound 15 and $16(736 \mathrm{mg}, 1.8 \mathrm{mmol})$ was treated with $p$-methoxybenzyl chloride as described for the synthesis of compound $\mathbf{2}$ to yield $\mathbf{1 7}$ as white crystals $(501 \mathrm{mg}, 31 \%$ over two steps) and $\mathbf{1 8}$ as white crystals (535 $\mathrm{mg}, 33 \%$ over two steps). (Column chromatography: DCM/Ac 93:7).

(17) $[\alpha]_{\mathrm{D}}{ }^{25}+77.31\left(c 0.12, \mathrm{CHCl}_{3}\right), \mathrm{R}_{f} 0.62$ (DCM/EtOAc 96:4), mp 89-90 ${ }^{\circ} \mathrm{C} .{ }^{1} \mathrm{H}$ NMR (500 $\left.\mathrm{MHz}, \mathrm{CDCl}_{3}\right): \delta_{\mathrm{H}} 7.84-6.82\left(16 \mathrm{H}, \mathrm{m}\right.$, arom.), $5.45\left(1 \mathrm{H}, \mathrm{s}, \mathrm{H}_{\mathrm{ac}}\right), 4.98-4.86\left(2 \mathrm{H}, 2 \mathrm{~d}, \mathrm{NAP}-\mathrm{CH}_{2}\right)$, 4.83-4.62 (2H, 2d, PMB-CH $), 4.72\left(1 \mathrm{H}, \mathrm{d},{ }^{3} J_{1,2}=3.5 \mathrm{~Hz}, \mathrm{H}-1\right), 4.16-4.13(2 \mathrm{H}, \mathrm{m}), 4.07(1 \mathrm{H}, \mathrm{dd}$, $J=3.5 \mathrm{~Hz}, J=10.0 \mathrm{~Hz}), 3.99(1 \mathrm{H}, \mathrm{dd}, J=3.5 \mathrm{~Hz}, J=10.0 \mathrm{~Hz}), 3.91(1 \mathrm{H}, \mathrm{dd}, J=1.5 \mathrm{~Hz}, J=$ $11.5 \mathrm{~Hz}), 3.75\left(3 \mathrm{H}, \mathrm{s}, \mathrm{PMB}-\mathrm{OCH}_{3}\right), 3.48(1 \mathrm{H}, \mathrm{s}), 3.34\left(3 \mathrm{H}, \mathrm{s}, \mathrm{OCH}_{3}\right) .{ }^{13} \mathrm{C} \mathrm{NMR}(125.7 \mathrm{MHz}$, $\mathrm{CDCl}_{3}$ ): $\delta_{\mathrm{C}} 159.2-113.6$ (arom.), $100.9\left(\mathrm{C}_{\mathrm{ac}}\right), 99.4(\mathrm{C}-1), 75.8,75.0,74.8,62.3$ (C-2, C-3, C-4, C5), 73.3, $72.2\left(2 \times \mathrm{CH}_{2}\right), 69.2(\mathrm{C}-6), 55.3,55.1\left(2 \times \mathrm{OCH}_{3}\right)$. Anal. Calcd for $\mathrm{C}_{33} \mathrm{H}_{34} \mathrm{O}_{7}(542.62)$ : C, 73.04; H, 6.32. Found: C, 73.14; H, 6.33. MALDI-TOF $\mathrm{m} / \mathrm{z}$ calcd for $[\mathrm{M}+\mathrm{Na}]^{+}: 565.61$, Found: 565.49 .

(18) $[\alpha]_{\mathrm{D}}{ }^{25}+62.60\left(c 0.12, \mathrm{CHCl}_{3}\right), \mathrm{R}_{f} 0.66(\mathrm{DCM} / \mathrm{EtOAc} 96: 4), \mathrm{mp}$ 101-103 ${ }^{\circ} \mathrm{C} .{ }^{1} \mathrm{H}$ NMR (500 $\left.\mathrm{MHz}, \mathrm{CDCl}_{3}\right): \delta_{\mathrm{H}} 7.79-6.83\left(16 \mathrm{H}, \mathrm{m}\right.$, arom.), $5.44\left(1 \mathrm{H}, \mathrm{s}, \mathrm{H}_{\mathrm{ac}}\right), 5.02-4.67\left(4 \mathrm{H}, 4 \mathrm{~d}, \mathrm{NAP}-\mathrm{CH}_{2}\right.$, PMB-CH $), 4.77\left(1 \mathrm{H}, \mathrm{d},{ }^{3} J_{1,2}=3.5 \mathrm{~Hz}, \mathrm{H}-1\right), 4.15\left(1 \mathrm{H}, \mathrm{dd},{ }^{3} J_{5,6 \mathrm{a}}=1.5 \mathrm{~Hz},{ }^{2} J_{6 \mathrm{a}, 6 \mathrm{~b}}=12.5 \mathrm{~Hz}, \mathrm{H}-\right.$ 6a), $4.12(1 \mathrm{H}, \mathrm{d}, \mathrm{H}-4), 4.10\left(1 \mathrm{H}, \mathrm{dd},{ }^{3} J_{2,3}=10.0 \mathrm{~Hz}, \mathrm{H}-2\right), 3.98\left(1 \mathrm{H}, \mathrm{dd},{ }^{3} J_{3,4}=3.5 \mathrm{~Hz}, \mathrm{H}-3\right), 3.95$ $\left(1 \mathrm{H}, \mathrm{dd},{ }^{3} J_{5,6 \mathrm{~b}}=1.5 \mathrm{~Hz}, \mathrm{H}-6 \mathrm{~b}\right), 3.75\left(3 \mathrm{H}, \mathrm{s}, \mathrm{PMB}-\mathrm{OCH}_{3}\right), 3.52(1 \mathrm{H}, \mathrm{s}, \mathrm{H}-5), 3.37\left(3 \mathrm{H}, \mathrm{s}, \mathrm{OCH}_{3}\right)$. ${ }^{13} \mathrm{C} \mathrm{NMR}\left(125.7 \mathrm{MHz}, \mathrm{CDCl}_{3}\right): \delta_{\mathrm{C}} 159.1-113.6$ (arom.), $100.9\left(\mathrm{C}_{\mathrm{ac}}\right), 99.4(\mathrm{C}-1), 75.6,75.3,74.6$, 62.4 (C-2, C-3, C-4, C-5), 73.7, $71.6\left(2 \mathrm{x} \mathrm{CH}_{2}\right), 69.2$ (C-6), 55.4, 55.1 (2 x OCH 3$)$. Anal. Calcd for $\mathrm{C}_{33} \mathrm{H}_{34} \mathrm{O}_{7}$ (542.62): C, 73.04; $\mathrm{H}, 6.32$. Found: $\mathrm{C}, 72.90 ; \mathrm{H}, 6.34$. MALDI-TOF m/z calcd for $[\mathrm{M}+\mathrm{Na}]^{+}:$565.61, Found: 565.48 .

Methyl 4,6-O-benzylidene-2- $O$-(p-methoxybenzyl)- $\alpha$-D-galactopyranoside (19). Compound 17 (160 mg, $0.3 \mathrm{mmol}$ ) was hydrogenolysed as described for the synthesis of compound 11 to obtain 19 as white crystals (96 mg, 80\%). (Column chromatography: DCM/Ac 93:7). $[\alpha]_{\mathrm{D}}^{25}+$ $60.20\left(c 0.50, \mathrm{CHCl}_{3}\right), \mathrm{mp} .92-95{ }^{\circ} \mathrm{C} .{ }^{1} \mathrm{H} \mathrm{NMR}\left(360 \mathrm{MHz}, \mathrm{CDCl}_{3}\right): \delta_{\mathrm{H}} 7.50-6.85(9 \mathrm{H}, \mathrm{m}$, arom.), $5.54\left(1 \mathrm{H}, \mathrm{s}, \mathrm{H}_{\mathrm{ac}}\right), 4.74\left(1 \mathrm{H}, \mathrm{d},{ }^{3} J_{1,2}=4.0 \mathrm{~Hz}, \mathrm{H}-1\right), 4.74-4.57\left(2 \mathrm{H}, 2 \mathrm{~d}, \mathrm{PMB}-\mathrm{CH}_{2}\right), 4.26-4.21(2 \mathrm{H}$, $\mathrm{m}), 4.13-4.08(1 \mathrm{H}, \mathrm{m}), 4.05(1 \mathrm{H}, \mathrm{dd}, J=1.4 \mathrm{~Hz}, J=12.5 \mathrm{~Hz}), 3.82-3.79(4 \mathrm{H}, \mathrm{m}), 3.65(1 \mathrm{H}, \mathrm{s})$, $3.36\left(3 \mathrm{H}, \mathrm{s}, \mathrm{OCH}_{3}\right), 2.44(1 \mathrm{H}, \mathrm{s}, \mathrm{OH}) .{ }^{13} \mathrm{C} \mathrm{NMR}\left(90 \mathrm{MHz}, \mathrm{CDCl}_{3}\right): \delta_{\mathrm{C}} 159.3-113.8$ (arom.),

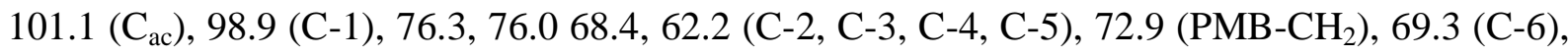
55.5, $55.2\left(2 \times \mathrm{OCH}_{3}\right)$. Anal. Calcd for $\mathrm{C}_{22} \mathrm{H}_{26} \mathrm{O}_{7}$ (402.44): C, 65.66; H, 6.51. Found: C, 65.90; $\mathrm{H}, 6.50$. MALDI-TOF m/z calcd for $[\mathrm{M}+\mathrm{Na}]^{+}: 425.43$, Found: 425.42.

Methyl 4,6- $O$-benzylidene-3- $O$-( $p$-methoxybenzyl)- $\alpha$-D-galactopyranoside (20). Compound 18 (160 mg, $0.3 \mathrm{mmol}$ ) was hydrogenolysed as described for the synthesis of $\mathbf{1 1}$ to obtain $\mathbf{2 0}$ as white crystals $(105 \mathrm{mg}, 88 \%)$. (Column chromatography: DCM/Ac 93:7). $[\alpha]_{\mathrm{D}}{ }^{25}+176.87(c 0.15$, $\left.\mathrm{CHCl}_{3}\right), \mathrm{mp} .175-176^{\circ} \mathrm{C} .{ }^{1} \mathrm{H} \mathrm{NMR}\left(360 \mathrm{MHz}, \mathrm{CDCl}_{3}\right): \delta_{\mathrm{H}} 7.53-6.84(9 \mathrm{H}, \mathrm{m}, \mathrm{arom}),. 5.45(1 \mathrm{H}, \mathrm{s}$, $\left.\mathrm{H}_{\mathrm{ac}}\right), 4.94\left(1 \mathrm{H}, \mathrm{d},{ }^{3} J_{1,2}=3.6 \mathrm{~Hz}, \mathrm{H}-1\right), 4.69-4.62\left(2 \mathrm{H}, 2 \mathrm{~d}, \mathrm{PMB}-\mathrm{CH}_{2}\right), 4.25\left(1 \mathrm{H}, \mathrm{dd},{ }^{3} J_{5,6 \mathrm{a}}=1.5\right.$ $\left.\mathrm{Hz},{ }^{2} J_{6 \mathrm{a}, 6 \mathrm{~b}}=12.4 \mathrm{~Hz}, \mathrm{H}-6 \mathrm{a}\right), 4.21-4.17(2 \mathrm{H}, \mathrm{m}, \mathrm{H}-2, \mathrm{H}-4), 4.02\left(1 \mathrm{H}, \mathrm{dd},{ }^{3} J_{5,6 \mathrm{~b}}=1.5 \mathrm{~Hz}, \mathrm{H}-6 \mathrm{~b}\right)$, 
$3.79\left(3 \mathrm{H}, \mathrm{s}, \mathrm{PMB}-\mathrm{OCH}_{3}\right), 3.77\left(1 \mathrm{H}, \mathrm{dd},{ }^{3} J_{2,3}=10.0 \mathrm{~Hz}, \mathrm{H}-3\right), 3.60(1 \mathrm{H}, \mathrm{s}, \mathrm{H}-5), 3.44(3 \mathrm{H}, \mathrm{s}$, $\left.\mathrm{OCH}_{3}\right), 2.30(1 \mathrm{H}, \mathrm{s}, \mathrm{OH}) .{ }^{13} \mathrm{C} \mathrm{NMR}\left(90 \mathrm{MHz}, \mathrm{CDCl}_{3}\right)$ : $\delta_{\mathrm{C}} 159.3-113.8$ (arom.), $100.9\left(\mathrm{C}_{\mathrm{ac}}\right), 99.9$

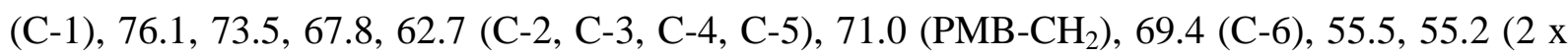
$\mathrm{OCH}_{3}$ ). Anal. Calcd for $\mathrm{C}_{22} \mathrm{H}_{26} \mathrm{O}_{7}$ (402.44): C, 65.66; H, 6.51. Found: C, 65.49; H, 6.49. MALDI-TOF m/z calcd for $[\mathrm{M}+\mathrm{Na}]^{+}: 425.43$, Found: 425.43 .

Methyl 2- $O$-acetyl-4,6- $O$-benzylidene-3- $O$-( $(p$-methoxybenzyl)- $\alpha$-D-galactopyranoside (21). To a stirred solution of compound $20(50 \mathrm{mg}, 0.15 \mathrm{mmol})$ in pyridine $(1 \mathrm{~mL}) \mathrm{Ac}_{2} \mathrm{O}(0.2 \mathrm{~mL})$ was added. When the TLC showed complete conversion, the solvent was evaporated, co-evaporated twice with toluene and the residue was purified by column chromatography (DCM/acetone 95:5) to yield 21 as a syrup $(51 \mathrm{mg}, 92 \%)$. $[\alpha]_{\mathrm{D}}{ }^{25}+15.31(c 0.11, \mathrm{MeOH}) .{ }^{1} \mathrm{H}$ NMR $(500 \mathrm{MHz}$, $\left.\mathrm{CDCl}_{3}\right): \delta_{\mathrm{H}} 7.54-6.84(9 \mathrm{H}, \mathrm{m}$, arom. $), 5.47\left(1 \mathrm{H}, \mathrm{s}, \mathrm{H}_{\mathrm{ac}}\right), 5.31\left(1 \mathrm{H}, \mathrm{dd},{ }^{3} J_{2,3}=10.5 \mathrm{~Hz}, \mathrm{H}-2\right), 5.06$ $\left(1 \mathrm{H}, \mathrm{d},{ }^{3} J_{1,2}=3.4 \mathrm{~Hz}, \mathrm{H}-1\right), 4.67-4.59\left(2 \mathrm{H}, 2 \mathrm{~d}, \mathrm{PMB}-\mathrm{CH}_{2}\right), 4.24\left(1 \mathrm{H}, \mathrm{d},{ }^{2} J_{6 \mathrm{a}, 6 \mathrm{~b}}=12.4 \mathrm{~Hz}, \mathrm{H}-6 \mathrm{a}\right)$, $4.16\left(1 \mathrm{H}, \mathrm{d},{ }^{3} J_{3,4}=3.2 \mathrm{~Hz}, \mathrm{H}-4\right), 4.01(1 \mathrm{H}, \mathrm{d}, \mathrm{H}-6 \mathrm{~b}), 3.96(1 \mathrm{H}, \mathrm{dd}, \mathrm{H}-3), 3.79$ (3H, s, PMB$\left.\mathrm{OCH}_{3}\right), 3.59(1 \mathrm{H}, \mathrm{s}, \mathrm{H}-5), 3.38\left(3 \mathrm{H}, \mathrm{s}, \mathrm{OCH}_{3}\right), 2.10\left(3 \mathrm{H}, \mathrm{s}, \mathrm{CH}_{3}\right) .{ }^{13} \mathrm{C} \mathrm{NMR}(125.7 \mathrm{MHz}$, $\left.\mathrm{CDCl}_{3}\right): \delta_{\mathrm{C}} 170.2(\mathrm{CO}), 159.2-113.7$ (arom.), $101.0\left(\mathrm{C}_{\mathrm{ac}}\right), 97.9(\mathrm{C}-1), 74.3,73.1,70.1,62.4(\mathrm{C}-2$, C-3, C-4, C-5), $71.5\left(\mathrm{PMB}-\mathrm{CH}_{2}\right), 69.2(\mathrm{C}-6), 55.4,55.2\left(2 \times \mathrm{OCH}_{3}\right), 21.0\left(\mathrm{CH}_{3}\right)$. Anal. Calcd for $\mathrm{C}_{24} \mathrm{H}_{28} \mathrm{O}_{8}$ (444.47): C, 64.85; H, 6.35. Found: C, 64.71; H, 6.37.

3,4-O-Isopropylidene-6- $O$-(methoxydimethyl)methyl-2- $O$-(2-naphthyl)methyl- $\beta$-Dgalactopyranosyl-(1 $\rightarrow$ 6)-1,2:3,4-di- $O$-isopropylidene- $\alpha$-D-galactopyranose $(23)$. To a stirred solution of compound $\mathbf{2 2}^{17}$ (1.3 g, $\left.2.4 \mathrm{mmol}\right)$ in DMF (10 mL) 60\% NaH (150 mg, 2 equiv) was added at $0^{\circ} \mathrm{C}$. After $30 \mathrm{~min}$ 2-(bromomethyl)naphthalene (540 mg, 1.3 equiv) was added to the reaction mixture. When TLC (DCM/acetone 75:25+1\% $\left.\mathrm{Et}_{3} \mathrm{~N}\right)$ showed complete conversion, it was diluted with methanol and evaporated. Usual work-up procedure resulted in compound $\mathbf{2 3}$ as a syrup $(1.55 \mathrm{~g}, 94 \%) \cdot[\alpha]_{\mathrm{D}}{ }^{25}+0.63\left(c 0.53, \mathrm{CHCl}_{3}\right) .{ }^{1} \mathrm{H} \mathrm{NMR}\left(360 \mathrm{MHz}, \mathrm{CDCl}_{3}\right): \delta_{\mathrm{H}} 7.91-7.42$ (7H, arom.), $5.60(1 \mathrm{H}, \mathrm{d}, J=5.1 \mathrm{~Hz}, \mathrm{H}-1), 5.07-5.00\left(2 \mathrm{H}, \mathrm{m}, \mathrm{NAP}-\mathrm{CH}_{2}\right), 4.61-4.59(1 \mathrm{H}, \mathrm{m})$, $4.40\left(1 \mathrm{H}, \mathrm{d}, J=8.1 \mathrm{~Hz}, \mathrm{H}-1^{\prime}\right), 4.35-4.33(1 \mathrm{H}, \mathrm{m}), 4.26-4.24(1 \mathrm{H}, \mathrm{m}), 4.17-4.11(4 \mathrm{H}, \mathrm{m}), 3.82-$ $3.65(4 \mathrm{H}, \mathrm{m}), 3.45-3.40(1 \mathrm{H}, \mathrm{m}), 3.21\left(3 \mathrm{H}, \mathrm{s}, \mathrm{OCH}_{3}\right), 1.55,1.46,1.35,1.34,1.33,1.32,1.29$, $1.20\left(24 \mathrm{H}, 8 \times \mathrm{x} \mathrm{s}, 8 \times \mathrm{CH}_{3}\right) .{ }^{13} \mathrm{C} \mathrm{NMR}\left(90 \mathrm{MHz}, \mathrm{CDCl}_{3}\right): \delta_{\mathrm{C}} 136.0-125.5$ (arom.), 109.6, 109.2,

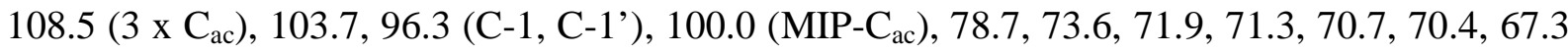
(skeleton carbons), 73.3 ( $\left.{ }^{\mathrm{NAP}}-\mathrm{CH}_{2}\right), 69.2,60.1$ (C-6, C-6'), $48.5\left(\mathrm{MIP}-\mathrm{OCH}_{3}\right), 27.6,26.3,26.0$, 25.9, 24.9, 24.3, 24.2 (6 x CH$)$. Anal. Calcd for $\mathrm{C}_{36} \mathrm{H}_{50} \mathrm{O}_{12}$ (674.77): C, 64.08; H, 7.47. Found: C, 64.17; H, 7.44.

\section{3,4- $O$-Isopropylidene-2- $O$-(2-naphthyl)methyl- $\beta$-D-galactopyranosyl-(1 $\rightarrow 6)-1,2: 3,4-d i-O$ -} isopropylidene- $\alpha$-D-galactopyranose (24). Compound $23(1.038 \mathrm{~g}, 1.5 \mathrm{mmol})$ was dissolved in DCM $(25 \mathrm{~mL})$ and $96 \% \mathrm{AcOH}(1 \mathrm{~mL})$ and 1 drop of water were added and it was stirred for $3 \mathrm{~h}$ at reflux temperature. After complete conversion of the starting material it was diluted with DCM $(60 \mathrm{~mL})$, washed with satd. aq. $\mathrm{NaHCO}_{3}$ and water, dried, filtered and evaporated. Purification by column chromatography resulted in compound 24 as white crystals $(0.67 \mathrm{~g}, 73 \%)$. [ $\alpha]_{\mathrm{D}}{ }^{25}$ +1.59 ( $c$ 0.16, $\mathrm{CHCl}_{3}$ ). mp:154-156 ${ }^{\circ} \mathrm{C} .{ }^{1} \mathrm{H} \mathrm{NMR}\left(360 \mathrm{MHz}, \mathrm{CDCl}_{3}\right): \delta_{\mathrm{H}} 7.88-7.43$ (7H, arom.), $5.59(1 \mathrm{H}, \mathrm{d}, J=5.1 \mathrm{~Hz}, \mathrm{H}-1), 5.04-5.00\left(2 \mathrm{H}, \mathrm{m}, \mathrm{NAP}-\mathrm{CH}_{2}\right), 4.64-4.61(1 \mathrm{H}, \mathrm{dd}), 4.42(1 \mathrm{H}, \mathrm{d}, J=$ 
8.1 Hz, H-1'), 4.35-4.29 (2H, m), 4.21-4.07 (4H, m), 3.95-3.90 (1H, m), 3.83-3.76 (3H, m),3.15$3.12(1 \mathrm{H}, \mathrm{m}), 3.53(1 \mathrm{H}, \mathrm{s}, \mathrm{OH}), 1.56,1.49,1.34,1.30,1.21\left(18 \mathrm{H}, 5 \mathrm{x} \mathrm{s}, 6 \mathrm{x} \mathrm{CH}_{3}\right) .{ }^{13} \mathrm{C} \mathrm{NMR}(90$ $\mathrm{MHz}, \mathrm{CDCl}_{3}$ ): $\delta_{\mathrm{C}} 135.8-125.8$ (arom.), 110.1, 109.4, 108.6 (3 $\mathrm{x} \mathrm{C}_{\mathrm{ac}}$ ), 103.4, $96.4(\mathrm{C}-1, \mathrm{C}-1$ '), 79.0, 78.6, 73.9, 73.0, 71.1, 70.7, 70.4, 67.1 (skeleton carbons), $73.3\left(\mathrm{NAP}-\mathrm{CH}_{2}\right), 68.9(\mathrm{C}-6)$, 62.4 (C-6'), 27.6, 26.3, 26.0, 25.9, 24.9, 24.3 (8 x CH 3 ). Anal. Calcd for $\mathrm{C}_{32} \mathrm{H}_{42} \mathrm{O}_{11}$ (602.67): C, 63.77; H, 7.02. Found: C, 63.97; H, 7.03.

3,4- $O$-Isopropylidene-6- $O$-( $p$-methoxybenzyl)-2- $O$-(2-naphthyl)methyl- $\beta$-D-galactopyranosyl$(\mathbf{1} \rightarrow \mathbf{6})$-1,2:3,4-di- $\boldsymbol{O}$-isopropylidene- $\boldsymbol{\alpha}$-D-galactopyranose (25). Compound 24 (380 mg, 0.63 mmol) was treated with $p$-methoxybenzyl chloride as described for the synthesis of compound 2 to yield 25 as a syrup (382 mg, 84\%). (Column chromatography $n$-hexane/acetone $75: 25)$. $[\alpha]_{\mathrm{D}}{ }^{25}$ $+0.46\left(c 0.19, \mathrm{CHCl}_{3}\right) .{ }^{1} \mathrm{H}$ NMR $\left(360 \mathrm{MHz}, \mathrm{CDCl}_{3}\right): \delta_{\mathrm{H}} 7.88-6.84(11 \mathrm{H}$, arom. $), 5.59(1 \mathrm{H}, \mathrm{d}, J=$ $5.1 \mathrm{~Hz}, \mathrm{H}-1), 5.06-5.00\left(2 \mathrm{H}, \mathrm{m}, \mathrm{NAP}-\mathrm{CH}_{2}\right), 4.59-4.47(3 \mathrm{H}, \mathrm{m}), 4.40(1 \mathrm{H}, \mathrm{d}, J=8.0 \mathrm{~Hz}, \mathrm{H}-1$ ' $)$, 4.14-4.10 (6H, m), 3.89-3.85 (1H, m), $3.77\left(3 \mathrm{H}, \mathrm{s}, \mathrm{OCH}_{3}\right), 3.75-3.70(3 \mathrm{H}, \mathrm{m}), 3.40-3.38(1 \mathrm{H}, \mathrm{m})$, $1.55,1.44,1.32,1.30,1.29,1.17\left(18 \mathrm{H}, 6 \mathrm{x} \mathrm{s}, 6 \times \mathrm{CH}_{3}\right) .{ }^{13} \mathrm{C} \mathrm{NMR}\left(90 \mathrm{MHz}, \mathrm{CDCl}_{3}\right): \delta_{\mathrm{C}} 159.1-$ 113.6 (arom.), 109.7, 109.3, 108.7 (3 x C $\mathrm{ac}$ ), 103.7, 96.3 (C-1, C-1'), 78.6, 78.5, 73.6, 71.9, 71.3, 70.6, 70.3, 67.4 (skeleton carbons), 73.2, $73.1\left(2 \mathrm{x} \mathrm{CH}_{2}\right), 68.5,67.9$ (C-6, C-6'), $55.1\left(\mathrm{OCH}_{3}\right)$, 27.6, 26.3, 26.0, 25.9, 24.9, $24.3\left(6 \times \mathrm{CH}_{3}\right)$. Anal. Calcd for $\mathrm{C}_{40} \mathrm{H}_{50} \mathrm{O}_{12}$ (722.82): C, 66.47; H, 6.97. Found: C, 66.24; H, 6.99.

3,4-O-Isopropylidene-6- $O$ - $(p$-methoxybenzyl)- $\beta$-D-galactopyranosyl-(1 $\rightarrow 6)-1,2: 3,4-d i-O$ isopropylidene- $\boldsymbol{\alpha}$-D-galactopyranose (26). Compound $25(700 \mathrm{mg}, 1.05 \mathrm{mmol})$ was hydrogenolysed as described for the synthesis of compound $\mathbf{1 1}$ to obtain $\mathbf{2 6}$ as a syrup (441 $\mathbf{m g}$, 71\%). (Column chromatography: DCM/acetone 9:1). $[\alpha]_{\mathrm{D}}{ }^{25}+0.51\left(c 0.16, \mathrm{CHCl}_{3}\right) .{ }^{1} \mathrm{H} \mathrm{NMR}$ (500 MHz, $\left.\mathrm{CDCl}_{3}\right): \delta_{\mathrm{H}} 7.27-6.86(4 \mathrm{H}$, arom.), $5.53(1 \mathrm{H}, \mathrm{d}, J=5.5 \mathrm{~Hz}, \mathrm{H}-1), 4.59-4.47$ (3H, m), 4.31-4.20 (3H, m), 4.14-4.12 (1H, m), 4.07-4.02 (3H, m), 4.00-3.91 $(1 \mathrm{H}, \mathrm{m}), 3.79\left(3 \mathrm{H}, \mathrm{s}, \mathrm{OCH}_{3}\right)$, $3.78-3.72(3 \mathrm{H}, \mathrm{m}), 3.58-3.55(1 \mathrm{H}, \mathrm{m}), 3.20(1 \mathrm{H}, \mathrm{s}, \mathrm{OH}), 1.52,1.50,1.43,1.33,1.31,1.20(18 \mathrm{H}$, $\left.6 \times \mathrm{x} \mathrm{s}, 6 \times \mathrm{CH}_{3}\right) .{ }^{13} \mathrm{C} \mathrm{NMR}\left(125.7 \mathrm{MHz}, \mathrm{CDCl}_{3}\right): \delta_{\mathrm{C}} 159.1,129.9,129.2,113.4$ (arom.), 109.8, 109.3, 108.6 (3 x C $\mathrm{ac}$ ), 103.3, 96.1 (C-1, C-1'), 78.7, 73.5, 73.2, 72.5, 71.1, 70.6, 70.3, 67.8 (skeleton carbons), 73.1 (PMB-CH$), 69.1,68.9$ (C-6, C-6'), $55.1\left(\mathrm{OCH}_{3}\right), 28.0,26.2,25.9,25.8$, $24.8\left(6 \times \mathrm{CH}_{3}\right)$. Anal. Calcd for $\mathrm{C}_{29} \mathrm{H}_{42} \mathrm{O}_{12}$ (582.64): C, 59.78; H, 7.27. Found: C, 59.88; H, 7.30.

p-Methoxyphenyl 2,6-di- $O$-benzyl-4- $O$-(p-methoxybenzyl)-3- $O$-(2-naphthyl)methyl-B-Dgalactopyranosyl-2,3,6-tri- $\boldsymbol{O}$-benzyl-ß)-D-glucopyranoside (28). Compound $27^{18}$ (234 mg, 0.23 mmol) was treated with $p$-methoxybenzyl chloride as described for the synthesis of compound 2 to yield 28 as a syrup (234 mg, 92\%). (Column chromatography: $n$-hexane/EtOAc 8:2). $[\alpha]_{\mathrm{D}}{ }^{25}$ $+1.54\left(c\right.$ 0.13, $\left.\mathrm{CHCl}_{3}\right) .{ }^{1} \mathrm{H} \mathrm{NMR}\left(360 \mathrm{MHz}, \mathrm{CDCl}_{3}\right): \delta_{\mathrm{H}} 7.77-6.76(40 \mathrm{H}$, arom.), 5.07-4.75 (10H, $\mathrm{m}), 4.56-4.23(6 \mathrm{H}, \mathrm{m}), 3.99-3.94(2 \mathrm{H}, \mathrm{m}), 3.80-3.37(16 \mathrm{H}, \mathrm{m}) .{ }^{13} \mathrm{C} \mathrm{NMR}\left(90 \mathrm{MHz}, \mathrm{CDCl}_{3}\right): \delta_{\mathrm{C}}$ 158.9-113.5 (arom.), 102.8, 102.7 (C-1, C-1'), 82.9, 82.4, 81.5, 79.9, 76.7, 75.3, 72.9 (skeleton carbons), 75.4, 75.1, 74.2, 73.7, 72.4 (7 x CH$), 68.3,68.1$ (C-6, C-6'), 55.5, 55.2 (2 x OCH$)$. Anal. Calcd for $\mathrm{C}_{73} \mathrm{H}_{74} \mathrm{O}_{13}$ (1159.36): C, 75.63; H, 6.43. Found: C, 75.76; H, 6.41. MALDI-TOF $\mathrm{m} / \mathrm{z}$ calcd for $[\mathrm{M}+\mathrm{Na}]^{+}: 1181.50$, Found: 1181.77 . 
p-Methoxyphenyl 2,6-di- $O$-benzyl-4- $O$-(p-methoxybenzyl)-ß-D-galactopyranosyl-2,3,6-tri$\boldsymbol{O}$-benzyl-ß)-D-glucopyranoside (29). Compound 28 (110 $\mathrm{mg}, 0.01 \mathrm{mmol})$ was hydrogenolysed as described for the synthesis of compound 3 to obtain 29 as a syrup (16 mg, 17\%). (Column chromatography: DCM/acetone 97:3, $\left.\mathrm{R}_{f} 045\right) .{ }^{1} \mathrm{H} \mathrm{NMR}\left(360 \mathrm{MHz}, \mathrm{CDCl}_{3}\right): \delta_{\mathrm{H}} 7.31-6.77(33 \mathrm{H}$, arom.), 5.06-4.27 (14H, m), 4.01-3.39 (19H, m). ${ }^{13} \mathrm{C} \mathrm{NMR}\left(90 \mathrm{MHz}, \mathrm{CDCl}_{3}\right): \delta_{\mathrm{C}} 159.2-113.7$ (arom.), 102.7 (C-1, C-1'), 82.9, 81.5, 80.7, 76.6, 75.5, 75.3, 74.0, 73.2 (skeleton carbons), 75.4, 75.1, 75.0, 74.7, 73.3, 73.1 (6 x CH 2$), 68.3,67.9$ (C-6, C-6'), 55.6, 55.2 (2 x $\left.\mathrm{OCH}_{3}\right)$. Anal. Calcd for $\mathrm{C}_{62} \mathrm{H}_{66} \mathrm{O}_{13}$ (1019.18): C, 73.06; H, 6.53. Found: C, 73.16; H, 6.50. MALDI-TOF m/z calcd for $[\mathrm{M}+\mathrm{Na}]^{+}:$1041.44, Found: 1041.39 .

Another chromatographically uniform product with lower mobility (DCM/acetone 97:3, $\mathrm{R}_{f} 0.21$ ) was also isolated $(13 \mathrm{mg})$. MALDI-TOF $\mathrm{m} / \mathrm{z}$ calcd. for $[\mathrm{M}+\mathrm{Na}]^{+}$of mono-debenzylated derivative of 29: 951.40, found: 951.30. The NMR spectra showed a mixture of isomers possessing the PMB group.

\section{Acknowledgement}

The work is supported by the TÁMOP 4.2.1/B-09/1/KONV-2010-0007 project. The project is co-financed by the European Union and the European Social Fund.

\section{References and Notes}

1. (a) Greene, T. W.; Wuts, P. G. M. Protective Groups in Organic Synthesis; John Wiley and Sons, Inc.: New York, 1991. (b) Kociensky, P. J. Protecting Groups; Georg Thieme: Stuttgart, 2004.

2. Oikawa, Y.; Yoshioka, T.; Yonemitsu, O. Tetrahedron Lett. 1982, 23, 885.

3. Johansson, R.; Samuelsson, B. J. Chem. Soc. Perkin Trans I 1984, 2371.

4. Wright, J. A.; Yu, J.; Spencer, J. B. Tetrahedron Lett. 2001, 42, 4033.

5. Sarkar, A. K., Rostand, K. S.; Jain, R. K.; Matta, K. L.; Esko, J. D. J. Biol. Chem. 1997, 272, 25608.

6. Gaunt, M. J.; Yu, J.; Spencer, J. B. J. Org. Chem. 1998, 63, 4172.

7. Gaunt, M. J.; Boschetti, C. E.; Yu, J.; Spencer, J. B. Tetrahedron Lett. 1999, 40, 1803.

8. Papageorgiou, E. A.; Gaunt, M. J.; Yu, J.; Spencer, J. B. Org. Lett. 2000, 2, 1049.

9. Xia, J.; Abbas, S. A.; Locke, R. D.; Piskorz, C. F.; Alderfer, J. L.; Matta, K. L. Tetrahedron Lett. 2000, 41, 169.

10. Kulkarni, S. S.; Liu, Y-H.; Hung, S-C. J. Org. Chem. 2005, 70, 2808.

11. Daragics, K.; Fügedi, P. Tetrahedron Lett., 2009, 50, 2914.

12. Csávás, M.; Hadházi, Á.; Borbás, A.; Lipták, A.; Antus, S. 16th European Carbohydrate Symposium, Sorrento, Italy, July 3-7, 2011: Abstract No 32, p246. 
13. This observation is in good correlation with the results of Spencer et al. (see Ref. 6). They assumed, that substitution of the aromatic ring could have an adverse steric effect that would interfere with the planar geometry required for effective binding and thus reduce its affinity for the metal surface. However, the Spencer group investigated only the selective hydrogenolysis of NAP in the presence of benzyl ethers, esters or carbamates (See Ref 6-8).

14. Borbás, A.; Szabó, Z. B.; Szilágyi, L.; Bényei, A.; Lipták, A. Tetrahedron, 2002 58, 5723.

15. Wang, C-C.; Lee, J-C.; Luo, S-Y.; Fan, H-F.; Pai, C-L.; Yang, W-C.; Lu, L-D.; Hung, S-C. Angew. Chem. Int. Ed. 2002, 41, 2360.

16. Jenkins, D. J.; Potter, B. V. L. Carbohydr. Res. 1994, 265, 145.

17. Borbás, A.; Jánossy, L.; Lipták, A. Carbohydr. Res. 1999, 318, 98.

18. Borbás, A.; Csávás, M.; Szilágyi, L.; Májer, G.; Lipták, A. J. Carbohydr. Chem. 2004, 13, 133.

19. Sajiki, H.; Kuno, H.; Hirota, K. Tetrahedron Lett. 1997, 38, 399.

20. Sajiki, H.; Hirota, K. Chem. Pharm. Bull. 2003, 51, 320. 